Supporting Information

\title{
Confronting Neutrality: Maximizing Success in the Analysis of Transition- Metal Catalysts by MALDI Mass Spectrometry
}

\author{
Gwendolyn A. Bailey and Deryn E. Fogg* \\ Centre for Catalysis Research \& Innovation, Department of Chemistry and Biomolecular \\ Sciences, University of Ottawa, Ottawa, Ontario, Canada K1N 6N5 \\ *Corresponding author. Email: dfogg@uottawa.ca
}

\section{Table of Contents}

S1. Spectrometers and experimental parameters used....................................................... S2

$\mathrm{S} 2$. Additional examples showing impact of matrix $\varepsilon_{\mathrm{M}}$ on fragmentation. ............................S3

S3. Spectra showing aggressive decomposition by functionalized matrices........................... S4

S4. Spectra showing impact of laser beam profile on fragmentation. ................................... S5

S5. UV-Vis spectra of selected matrices and analytes................................................... S6 


\section{S1. Spectrometers and experimental parameters used.}

Table S1. MALDI mass spectrometers used, and relevant instrument parameters.

\begin{tabular}{|c|c|c|c|c|}
\hline \multirow[b]{2}{*}{ Instrument } & \multirow[b]{2}{*}{ Location } & \multicolumn{2}{|c|}{ Laser } & \multirow[b]{2}{*}{ Figure } \\
\hline & & Type & Wavelength & \\
\hline AB Sciex 5800 & MALDI MS / Functional & Nd:YLF & $349 \mathrm{~nm}$ & $3, \mathrm{~S} 3 \mathrm{a}$ \\
\hline TOF/TOF & $\begin{array}{l}\text { Proteomics Facility, } \\
\text { London Regional } \\
\text { Proteomics Centre, } \\
\text { Western Univ. }\end{array}$ & & & \\
\hline AB Sciex 5800 & AB Sciex & Nd:YLF & $349 \mathrm{~nm}$ & $5 \mathrm{e}$ \\
\hline TOF/TOF & Framingham, MA & & & \\
\hline $\begin{array}{l}\text { Applied Biosystems } \\
4700 \mathrm{TOF} / \mathrm{TOF}\end{array}$ & $\begin{array}{l}\text { MALDI MS / Functional } \\
\text { Proteomics Facility, } \\
\text { London Regional } \\
\text { Proteomics Centre, } \\
\text { Western Univ. }\end{array}$ & Nd:YAG & $355 \mathrm{~nm}$ & $\mathrm{~S} 4 \mathrm{a}^{a}$ \\
\hline Applied Biosystems & Univ. Toronto & Nd:YAG & $355 \mathrm{~nm}$ & $2,6,9 a$ \\
\hline $4800 \mathrm{TOF} / \mathrm{TOF}$ & Forestry Dept. & & & \\
\hline Bruker Microflex TOF & $\begin{array}{l}\text { Bruker Daltonics } \\
\text { Billerica MA }\end{array}$ & $\mathrm{N}_{2}$ & $337 \mathrm{~nm}$ & $\mathrm{~S} 3 \mathrm{c}$ \\
\hline Bruker Omniflex TOF & $\begin{array}{l}\text { Fogg Anaerobic MALDI- } \\
\text { MS Facility, Univ. } \\
\text { Ottawa }\end{array}$ & $\mathrm{N}_{2}$ & $337 \mathrm{~nm}$ & $\mathrm{~S} 3 \mathrm{c}$ \\
\hline Bruker Reflex IV TOF & $\begin{array}{l}\text { MALDI MS / Functional } \\
\text { Proteomics Facility } \\
\text { London Regional } \\
\text { Proteomics Centre, } \\
\text { Western Univ. }\end{array}$ & $\mathrm{N}_{2}$ & $337 \mathrm{~nm}$ & $\mathrm{~S} 4 \mathrm{~b}^{a}$ \\
\hline $\begin{array}{l}\text { Bruker Ultraflex II } \\
\text { TOF/TOF }\end{array}$ & $\begin{array}{l}\text { Bruker Daltonics } \\
\text { Billerica, MA }\end{array}$ & $\mathrm{N}_{2}$ & $337 \mathrm{~nm}$ & $9 b$ \\
\hline Bruker UltrafleXtreme & Bruker Daltonics & contoured & $355 \mathrm{~nm}$ & $1,4,5 \mathrm{a}-\mathrm{d}, 7$ \\
\hline $\mathrm{TOF} / \mathrm{TOF}$ & Billerica, MA & Nd:YAG & & 9c, S1, S2 \\
\hline Shimadzu Performance & Shimadzu & $\mathrm{N}_{2}$ & $337 \mathrm{~nm}$ & $\mathrm{~S} 3 \mathrm{c}$ \\
\hline TOF/TOF & Columbia MD & & & \\
\hline ThermoFisher MALDI & Laboratory of Imaging & $\mathrm{N}_{2}$ & $337 \mathrm{~nm}$ & 8 \\
\hline LTQ Orbitrap XL & $\begin{array}{l}\text { Mass Spectrometry } \\
\text { Univ. North Texas }\end{array}$ & & & \\
\hline $\begin{array}{l}\text { Waters Micro MX } \\
\text { TOF }\end{array}$ & $\begin{array}{l}\text { Advanced Instrumental } \\
\text { Mass Spectrometry Lab } \\
\text { Univ. Toronto }\end{array}$ & $\mathrm{N}_{2}$ & $337 \mathrm{~nm}$ & $\mathrm{~S} 3 \mathrm{c}$ \\
\hline $\begin{array}{l}\text { Waters Synapt G2-Si } \\
\text { MALDI-QTOF }\end{array}$ & $\begin{array}{l}\text { Waters Corp. } \\
\text { Milford MA }\end{array}$ & Nd:YAG & $355 \mathrm{~nm}$ & $\mathrm{~S} 3 \mathrm{~b}$ \\
\hline
\end{tabular}

${ }^{a}$ At the time of use, these lasers were near the end of their operational lifetimes, and higher applied laser energies were therefore required. The impact on fragmentation is illustrated in Fig. S4. 


\section{S2. Additional examples showing impact of matrix $\varepsilon_{M}$ on fragmentation.}

These spectra supplement the two extremes shown in Figure 4.

(a)

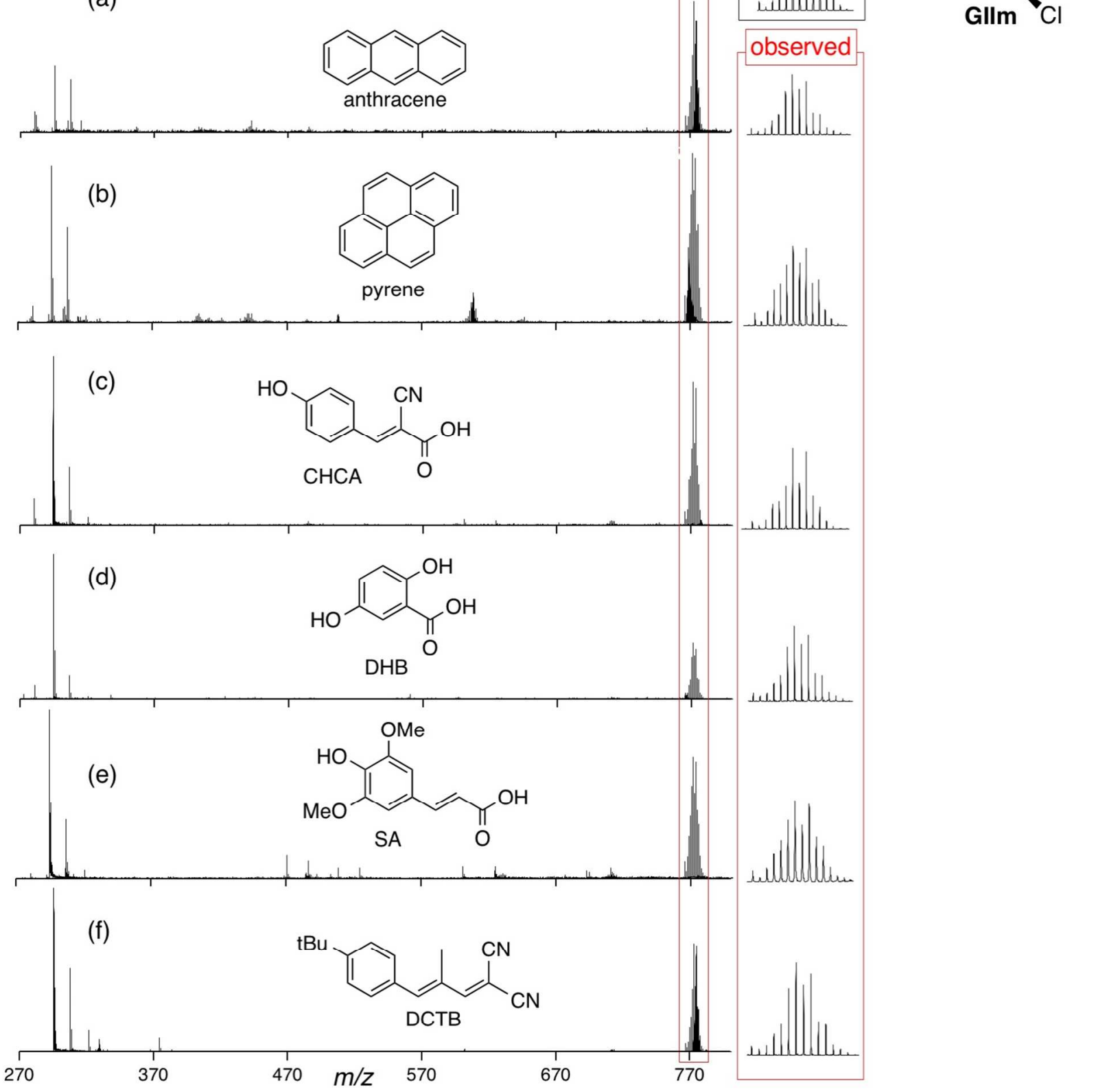

Figure S1. Impact of matrix $\varepsilon_{\mathrm{M}}$ on fragmentation, assessed in analysis of non-labile GIIm. MALDI mass spectra of GIIm with (a) anthracene; (b) pyrene; (c) CHCA; (d) DHB; (e) SA; (f) DCTB. 


\section{S3. Spectra showing aggressive decomposition by functionalized matrices.}

These examples supplement the spectra shown in Figure 5, which focus on more widely-used matrices.

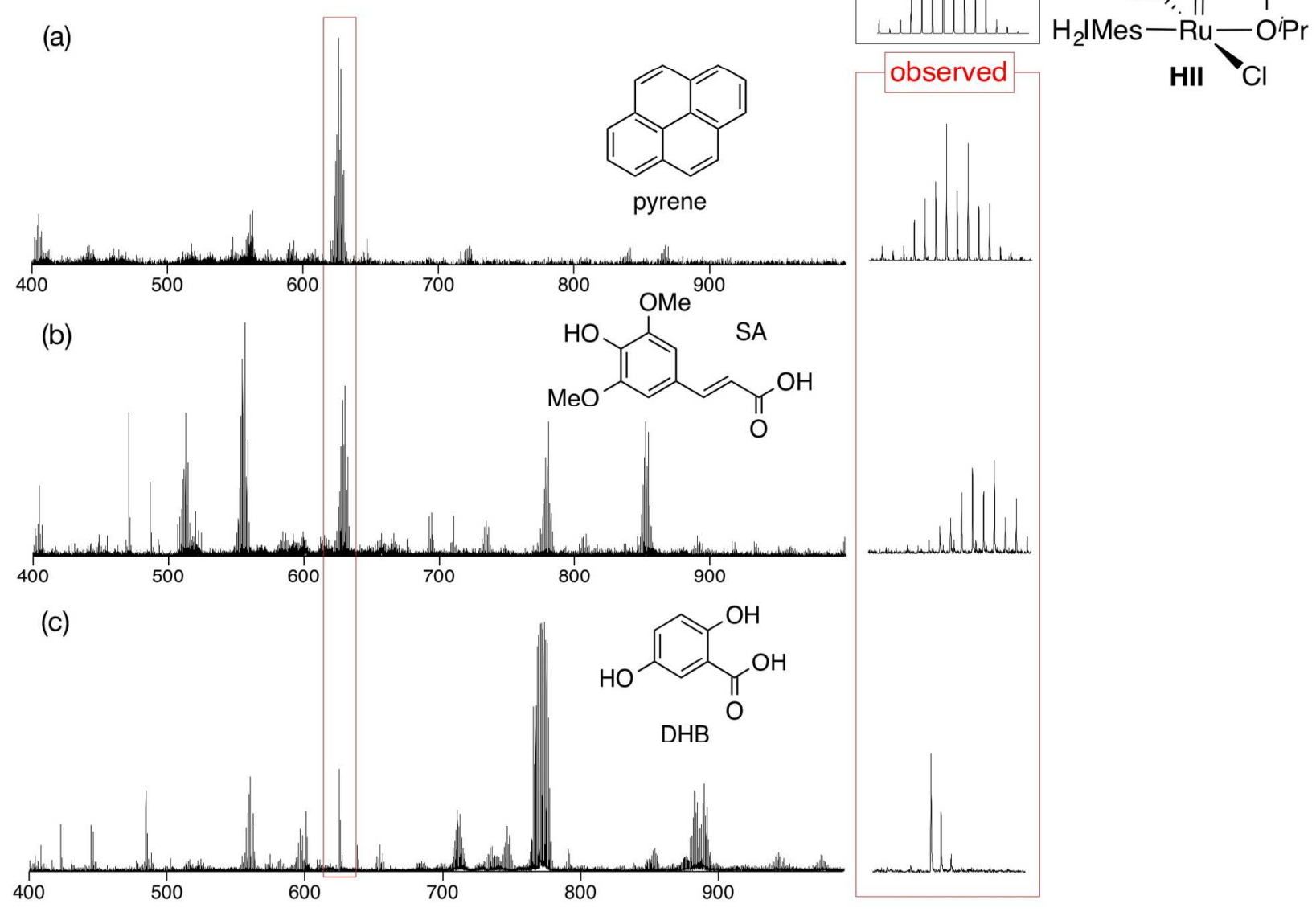

Figure S2. MALDI mass spectra showing gas-phase decomposition of HII by functionalized matrices, as compared to the benchmark pyrene. (a) Pyrene; (b) SA; (c) DHB. 


\section{S4. Spectra showing impact of laser beam profile on fragmentation.}

These examples supplement the spectra shown in Figure 9 (which were drawn from instruments matched as closely as possible, to facilitate comparison).
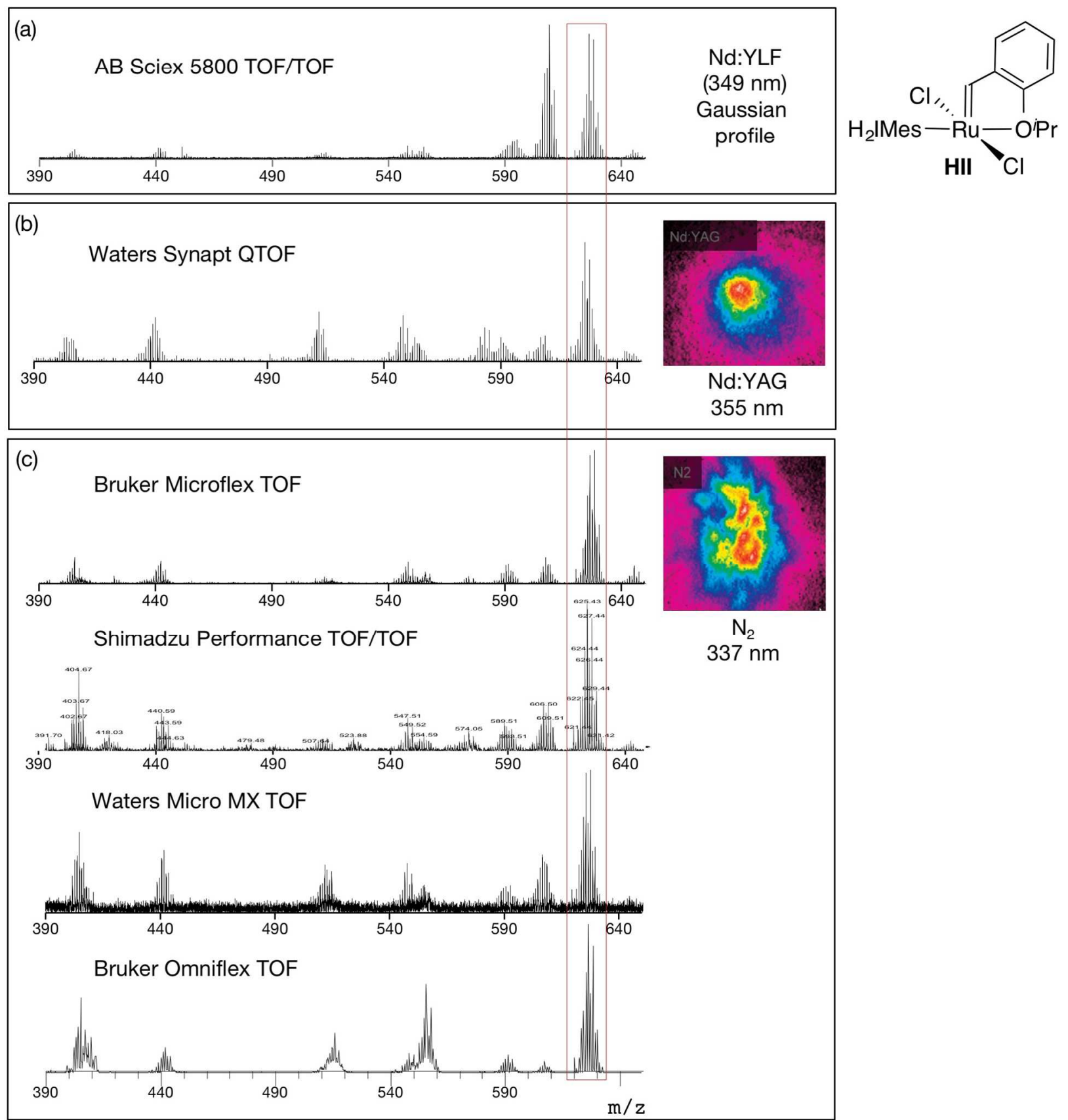

Figure S3. Impact of laser beam profile on fragmentation of HII. Spectra recorded using (a) a $\mathrm{Nd}$ :YLF laser; (b) a Nd:YAG laser; and (c) a $\mathrm{N}_{2}$ laser. 


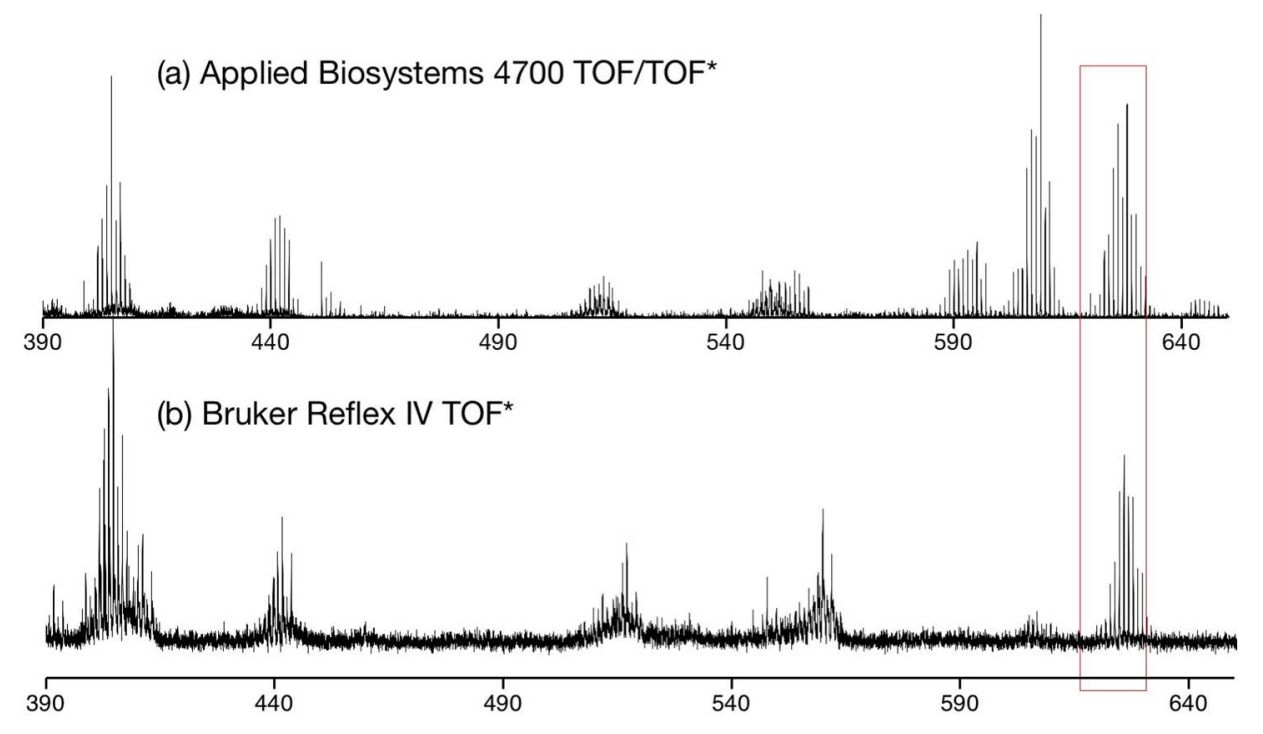

Figure S4. Negative impact of laser age on performance. MALDI mass spectra recorded for HII on (a) Nd:YAG (Applied Biosystems 4700) and (b) $\mathrm{N}_{2}$ (Bruker Reflex II) lasers that were nearing the end of their lifetime, necessitating use of higher applied laser energies.

\section{S5. UV-vis spectra of selected matrices and analytes.}
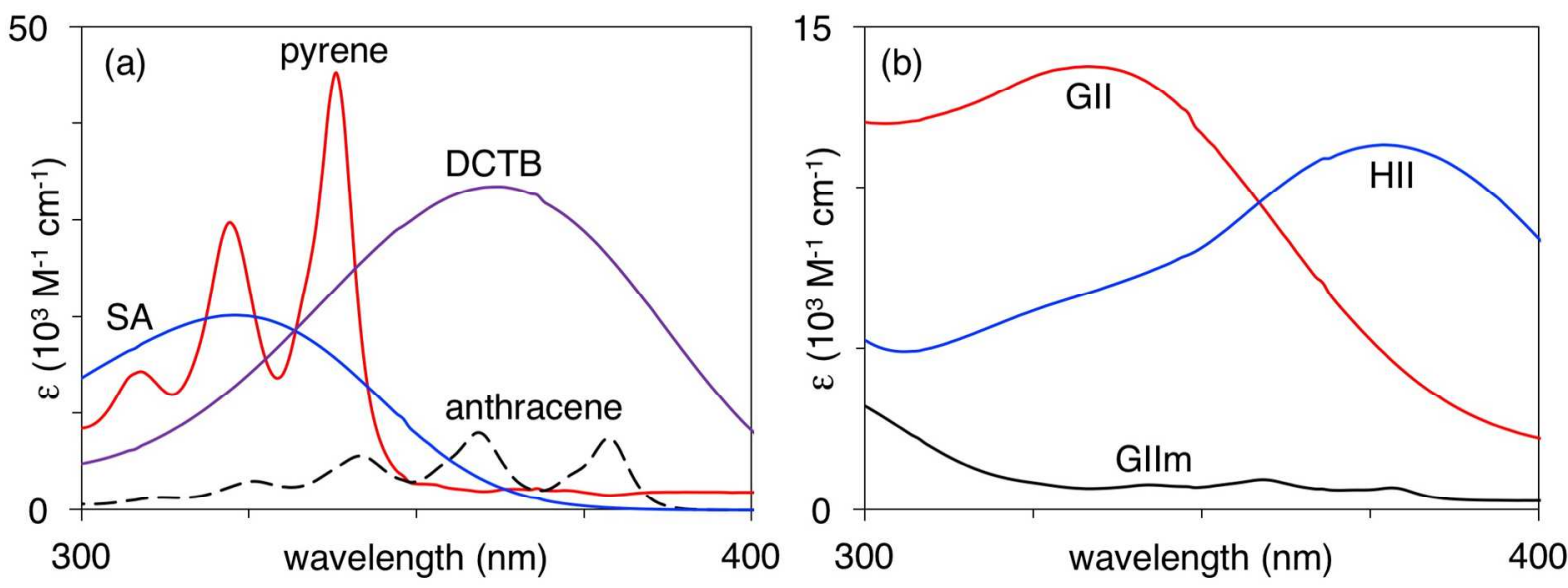

Figure S5. (a) UV-vis spectra of the matrices pyrene, anthracene, and DCTB in $\mathrm{CH}_{2} \mathrm{Cl}_{2}$; spectrum of SA (which is very poorly soluble in $\mathrm{CH}_{2} \mathrm{Cl}_{2}$ ) in methanol. (b) UV-vis spectra of the analytes GII, GIIm, and $\mathrm{HII}$ in $\mathrm{CH}_{2} \mathrm{Cl}_{2}$. 